\title{
Optimum Droplet Motion in Fire Plumes
}

\author{
V. Novozhilov \\ Fire Safety Engineering Research and Technology Centre \\ Built Environment Research Institute \\ University of Ulster, United Kingdom \\ E-mail: vb.novozhilov@ulster.ac.uk
}

\begin{abstract}
The present paper introduces analytical model applicable for analysis of motion of water droplets injected into fire-generated plumes. The model is derived from Lagrangian equation of droplet motion. Application of the developed model to the practically important problem that is fire suppression by water sprays is discussed. A criterion for optimum spray dynamics is proposed. An analytical expression is provided for the optimum droplet size in the spray as a function of Heat Release Rate (HRR) of fire. The present approach provides a quick estimation of optimum spray parameters for a particular fire suppression application.
\end{abstract}

Keywords: Droplet Dynamics, Fire Suppression, Lagrangian Model, Optimum Fire Fighting

\section{Introduction}

Droplet motion in flows of various configurations is of great theoretical and practical interest. Very little research is done, however, on this topic in terms of developing approximate analytical solutions. Morsi and Alexander [1] considered the problem of spherical particles movement in a one-dimensional and in some two-dimensional flows (around a circular cylinder and around a lifting aerofoil section). They were only able to solve equations analytically in the case of onedimensional flow. Recently, Novozhilov [2] presented analytical solutions for both non-evaporating and evaporating particles in the two-dimensional axisymmetrical case, with the flow parallel to the axis of symmetry and changing in the radial direction only.

The present paper concerns with the specific case of spherical droplet movement in a flow generated by fire plume. Flow is approximated as one- 
dimensional but its velocity profile behaves in a peculiar manner, specific for convective plumes.

The problem considered is of great practical interest for fire fighting which still mostly relies on fire suppression by different forms of water sprays. In particular, the effect of particle sixe is quite important. Droplet size distribution has a strong influence on spray/fire plume interaction; it controls the rate of spray evaporation in the flame and eventually the efficiency of flame suppression. These features have been extensively observed in fire suppression studies [3-5]. Fire suppression by water spray can occur in the two distinctive regimes [3]: via fuel cooling (surface suppression) or via flame cooling (gaseous suppression). First corresponds to relatively coarse sprinkler sprays, the second to fine water mists. The most efficient way of fire suppression is associated with the latter mode. These regimes are demonstrated qualitatively in Figure 1.

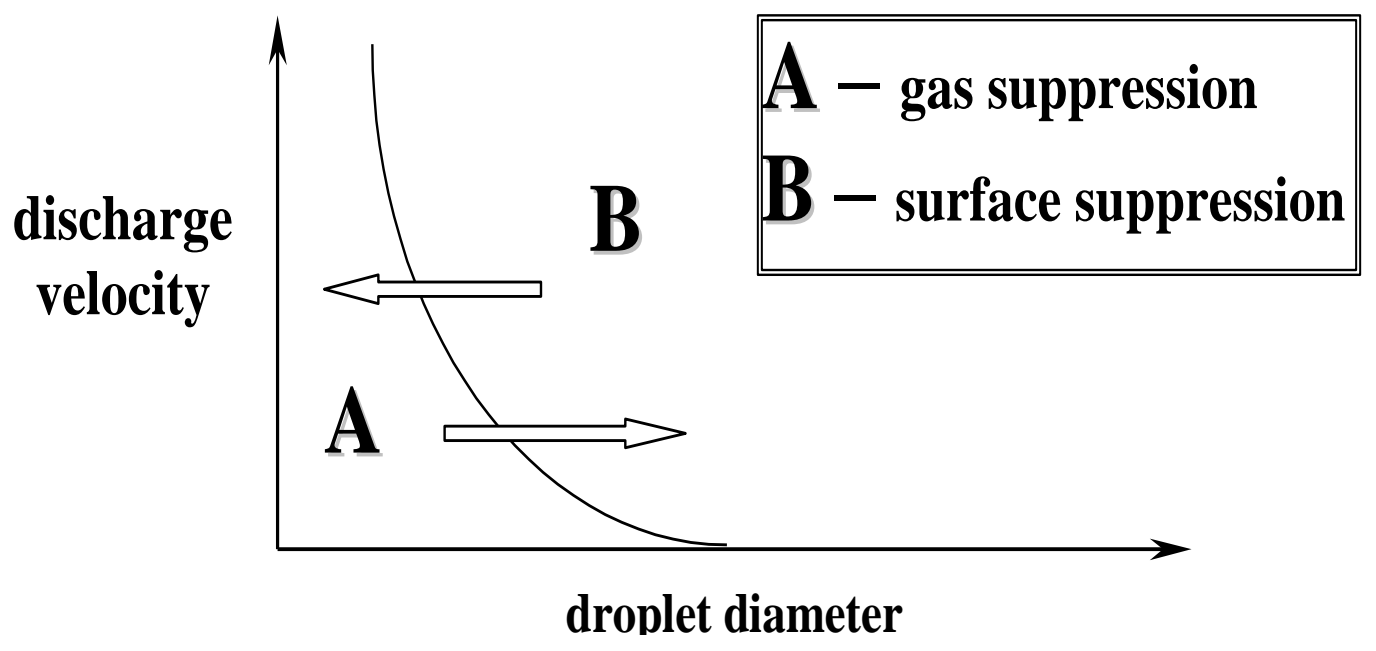

Figure 1 Schematic of suppression regimes

An efficient application of either of these strategies requires that the spray parameters are optimized to achieve maximum heat extraction rate. As explained above, the primary parameter that can be varied for such an optimization is droplet size.

Several studies [6-9] attempted to define optimum droplet size in a different manner, and obtained numerical estimations corresponding to their definitions. Criteria for optimality and predicted optimum droplet diameters vary wildly.

In the present study an entirely novel concept of optimum spray diameter is proposed. It is based on the strategy of flame suppression in the gaseous phase, and applies therefore, in general, to fire suppression regimes delivered by water mist systems. A distinguishing feature of this approach is that the estimation for 
the optimum diameter is derived from exact equation of droplet motion and exact flow velocity profile in fire plume and flame. Starting from exact equations, the model however provides surprisingly simple formulas for optimum droplet diameter in fire fighting sprays.

It is argued that a proper scientific definition of water mist sprays need be based on consideration of spray dynamics under specific fire conditions.

\section{Mathematical Model}

Consider the basics of water droplet interaction with flame and fire plume (Figure 2). Fire-induced flow structure consists of three distinctive zones: continuous flame zone, intermittent flame zone (where luminous flame is appearing only for fraction of time), and fire plume zone (convective flow of inert combustion products mixed with surrounding air).

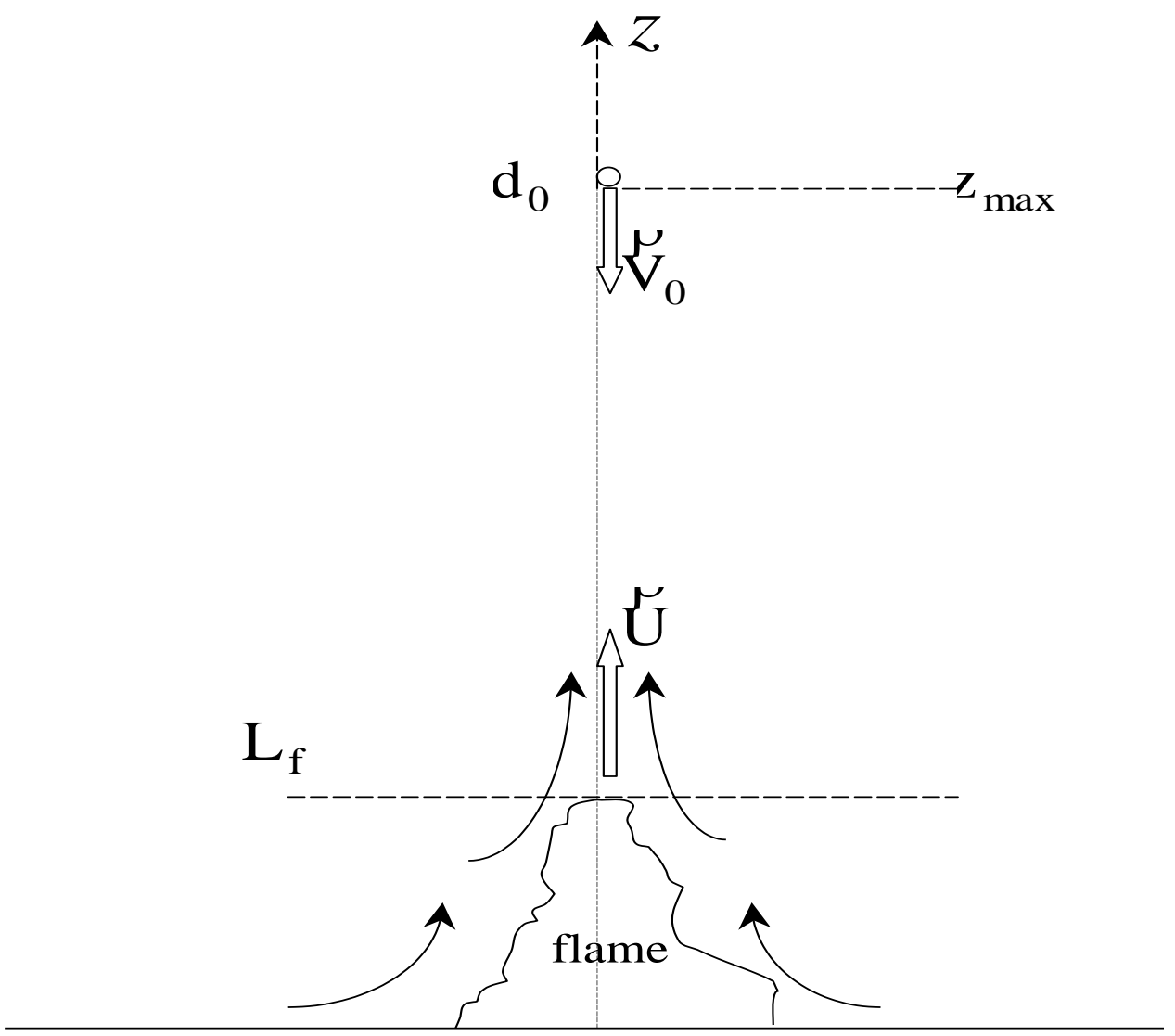

Figure 2 Schematic of droplet injection into fire plume

The equation of droplet motion is taken in the form [10] 


$$
\begin{aligned}
& m \frac{d \vec{V}}{d t}=-\frac{\pi}{8} d^{2} \rho C_{D}|\vec{V}-\vec{U}(z)|(\vec{V}-\vec{U}(z))+\vec{F}_{g} \\
& \text { or } \\
& \frac{d \vec{V}}{d t}=-\left(\frac{3}{4} \frac{\rho}{\rho_{p}}\right) \frac{C_{D}}{d}(\vec{V}-\vec{U}(z))|\vec{V}-\vec{U}(z)|+\vec{g}
\end{aligned}
$$

According to arrangements of Figure 2, components of the velocities obey $V<0 ; U>0 ; V-U<0$.

Taking this into account, the equation (2) in components becomes

$$
\frac{d V}{d t}=\left(\frac{3}{4} \frac{\rho}{\rho_{p}}\right) \frac{C_{D}}{d}(V-U(z))^{2}-g
$$

At this point, an approximation regarding the form of dependence of the drag coefficient on the particle Reynolds number is made. It is customary to characterize particle drag coefficient, for example, by the following correlation [11]

$C_{D}=\left\{\begin{array}{lr}27 / \mathrm{Re}_{p}^{0.84}, & \operatorname{Re}_{p}<80 \\ \hline 0.271 \operatorname{Re}_{p}^{0.217}, & 80<\operatorname{Re}_{p}<10^{4}\end{array}\right.$

In the present analysis, this dependence is replaced by the following simplifying one

$$
C_{D}=27 / \operatorname{Re}_{p}, \quad \operatorname{Re}_{p}<10^{4}
$$

It has been demonstrated [2] that the correlation (5) is quite reasonable, and certainly provides good practical results in the context of the present analysis. The use of a simple universal correlation (5) greatly simplifies the equation (3). Indeed, on the RHS of (3) one has

$\frac{C_{D}}{d}(V-U(z))^{2}=\frac{27 v(U(z)-V)^{2}}{d(U(z)-V) d}=27 v \frac{1}{d^{2}}(U(z)-V)$

Finally, the basic approximate equation of the droplet motion used throughout the present paper is

$$
\frac{d V}{d t}=\frac{\alpha}{d^{2}}(U(z)-V)-g
$$


with $\alpha=\frac{81}{4} v \frac{\rho}{\rho_{p}}$. The profile $U(z)$ in the above equation is assumed to be known, and is determined by a particular fire under consideration.

Even in the case of constant droplet diameter no analytical solutions for equation (7) are known, although the equation form itself is rather familiar. In the latter case (constant diameter) the equation (7) belongs to the class of Abel Equations of the Second Kind. Existence of closed-form solutions for these equations depend on the form of a known function on the RHS, in our case, essentially, on the form of the profile $U(z)$. Unfortunately, the case of velocity profiles in fire plumes $U(z) \sim z^{-1 / 3}$ does not fall into the classified cases which admit integration [12].

\section{Results and Discussion}

The basic idea of the analysis is that for the particular present application (optimum droplet movement), only the lowest point of the droplet trajectory is most important. This is explained in more detail below. This consideration allows the problem of non-integrable case of equation (7) to be circumvented. It will be demonstrated the closed model predicting the lowest point of the droplet trajectory can be obtained.

\subsection{Non-evaporating droplet}

To illustrate the method for estimating optimum diameter, consider movement of a non-evaporating particle first. This case illustrates essential properties of solution of the equation (7).

In general, the optimum should be defined in the following way: droplet posses the optimum diameter if upon injection it evaporates exactly in the flame region (i.e. neither penetrates to the surface nor evaporates in inert fire plume). In application to a non-evaporating particle, it is sensible to impose the following criterion: zero-velocity point of the droplet's trajectory is inside the flame. This point will also be a reversing point for a droplet trajectory, since upon achieving this point the droplet will still experience acceleration by fire plume, and will start to move upwards. The rationale for such a criterion is that if the droplet had a capability to evaporate, complete evaporation is likely to happen in the vicinity of a reversing point where the temperature is high (flame temperature) and droplet motion is slow (residence time large).

It will be demonstrated below that a similar, slightly modified criterion is suitable for a general case of evaporating droplet.

Using the identity 
$\frac{d V}{d t}=\frac{d V}{d z} \cdot \frac{d z}{d t}=V \frac{d V}{d z}$

the equation (7) is transformed into

$V \frac{d V}{d z}=\frac{\alpha}{d^{2}}(U(z)-V)-g$

This equation can now be integrated between the initial $\left(z_{\max }\right)$ and final $\left(z_{\min }\right)$ points of the trajectory (we remind that $d=$ const at the moment) to get

$$
-\frac{1}{2} V_{0}^{2}=\frac{\alpha}{d^{2}}\left[\int_{z_{\max }}^{z_{\min }} U(z) d z-\int_{z_{\max }}^{z_{\min }} V d z\right]-g\left(z_{\min }-z_{\max }\right)
$$

The integral term containing plume velocity profile $U(z)$ is a known function, obtained upon specification of the latter profile.

The term involving unknown profile of the particle velocity $V(z)$ needs, however, be estimated.

This integral term can be written (using (8) again) as

$$
\int_{z_{\max }}^{z_{\min }} V d z=\int_{z_{\max }}^{z_{\min }} V^{2} d t
$$

and the simplest estimation (mean value theorem) yields

$$
\int_{z_{\max }}^{z_{\min }} V^{2} d t \approx V\left(z_{*}\right) \int_{z_{\max }}^{z_{\min }} V d t=V\left(z_{*}\right)\left[z_{\text {min }}-z_{\text {max }}\right] \approx-\frac{V_{0} \mid}{2}\left[z_{\text {min }}-z_{\text {max }}\right]=\frac{\left|V_{0}\right|}{2}\left[z_{\text {max }}-z_{\text {min }}\right] .
$$

Therefore, the equation (10) gives

$$
\begin{aligned}
& -\frac{1}{2} V_{0}^{2}=\frac{\alpha}{d^{2}}\left[\int_{z_{\max }}^{z_{\min }} U(z) d t-\frac{V_{0} \mid}{2}\left(z_{\text {max }}-z_{\text {min }}\right)\right]-g\left(z_{\text {min }}-z_{\text {max }}\right) \\
& \frac{1}{d^{2}}\left[\int_{z_{\max }}^{z_{\min }} U(z) d z-\frac{\left|V_{0}\right|}{2}\left(z_{\text {max }}-z_{\text {min }}\right)\right]=\frac{1}{\alpha}\left[g\left(z_{\text {min }}-z_{\text {max }}\right)-\frac{1}{2} V_{0}^{2}\right]
\end{aligned}
$$


and

$d_{\text {opt }}^{2}=\alpha \frac{\left[\int_{z_{\max }}^{z_{\min }} U(z) d z-\frac{\left|V_{0}\right|}{2}\left(z_{\text {max }}-z_{\text {min }}\right)\right]}{\left[g\left(z_{\text {min }}-z_{\text {max }}\right)-\frac{1}{2} V_{0}^{2}\right]}$

Finally, one arrives at the estimation:

$d_{o p t}=\left[\alpha \frac{\int_{z_{\max }}^{z_{\min }} U(z) d z-\frac{\left|V_{0}\right|}{2}\left(z_{\text {max }}-z_{\text {min }}\right)}{g\left(z_{\text {min }}-z_{\text {max }}\right)-\frac{1}{2} V_{0}^{2}}\right]^{1 / 2}$

At this point, plume velocity profile must be specified. The reference fire used for illustration is a methanol pool fire, which exhibits nearly constant burning rate over a wide range of pool diameters.

For a particular fire HRR, a corresponding pool diameter can be found from the burning rate assuming complete combustion of the fuel. Then, flame heights, virtual origin position, and plume velocity profile can be predicted by well-known correlations of Heskestad [13].

The plume velocity profile is given as

$$
\begin{aligned}
& U(z)=\gamma\left(\frac{g}{c_{p} T_{\infty} \rho_{\infty}}\right)^{1 / 3} \dot{Q}_{c}^{1 / 3}\left(z-z_{0}\right)^{-1 / 3} \\
& \left(\gamma \approx \frac{34}{10}\right)
\end{aligned}
$$

Velocity distribution in the flame zone is different. Velocity is nearly constant in the intermittent zone, and falls to zero following square-root dependence in the continuous zone [13]. Intermittent zone typically covers about $60 \%$ of the total flame height [13]. Based on these estimations, velocity profile (17) can be extended into the flame zone as

$U(z)=\sqrt{\frac{5}{2}} U\left(L_{f}\right)\left(\frac{z}{L_{f}}\right)^{1 / 2} ; \quad z \leq \frac{2}{5} L_{f}$ 
$U(z)=U\left(L_{f}\right) ; \quad \frac{2}{5} L_{f} \leq z \leq L_{f}$

This procedure uniquely relates fire HRR to the plume geometry and thermal properties.

In the present example, half height of the flame is taken as a trajectory reverse point, i.e. $\quad z_{\min }=L_{f} / 2$. Upon integration of the profile (17-19), the following expression is obtained:

$$
\begin{aligned}
& \int_{z_{\max }}^{L_{f} / 2} U(z) d z=\gamma_{1}\left(\frac{g}{c_{p} T_{\infty} \rho_{\infty}}\right)^{1 / 3} \dot{Q}_{c}^{1 / 3}\left[\left(L_{f}-z_{0}\right)^{2 / 3}-\left(z_{\max }-z_{0}\right)^{2 / 3}\right]-\frac{1}{2} U\left(L_{f}\right) L_{f} \approx \\
& \approx\left(\frac{g}{c_{p} T_{\infty} \rho_{\infty}}\right)^{1 / 3} \dot{Q}_{c}^{1 / 3}\left[\gamma_{1}\left[\left(L_{f}-z_{0}\right)^{2 / 3}-\left(z_{\max }-z_{0}\right)^{2 / 3}\right]-\gamma_{2}\left(L_{f}-z_{0}\right)^{-1 / 3} L_{f}\right]
\end{aligned}
$$

$\left(\gamma_{1}=3 / 2 \gamma ; \gamma_{2}=1 / 2 \gamma\right)$

The equations $(16,20)$ provide required estimation for the optimum droplet diameter.

Quality of prediction is tested against numerical solution of the equation of droplet motion, coupled to the mass transfer equation. Description of the Lagrangian method of particle (droplet) tracking is widely available in the literature, e.g. $[3,4]$.

Droplet initial velocity is kept constant at $V_{0}=20 \mathrm{~m} / \mathrm{s}$. Initial droplet height is $3 \mathrm{~m}$ for the present case, and $5 \mathrm{~m}$ for the evaporating droplet analysis (below).

Prediction results for a non-evaporating droplet are presented in Figure 3. There is an excellent agreement between the analytical estimation $(16,20)$ and the solution obtained by numerical integration.

\subsection{Evaporating droplet}

Similar ideas are applicable for the droplet experiencing evaporation in fire plume and flame.

The optimum is redefined here in the following way: droplet trajectory ends at the surface of the material due to complete evaporation. This ensures, first of all, that droplet is delivered into the flame zone and completely evaporates in this zone, i.e. it is not being carried away by the plume. Secondly, major part of evaporation 
process is expected to occur in the flaming zone, since the droplet will significantly decelerate before it disappears at the surface. (Note that the point of complete evaporation is always also a point of zero velocity for the droplet trajectory). Therefore, the droplet will have large residence time in the flame. Strict mathematical proof of the above criterion is yet to follow in subsequent publications.

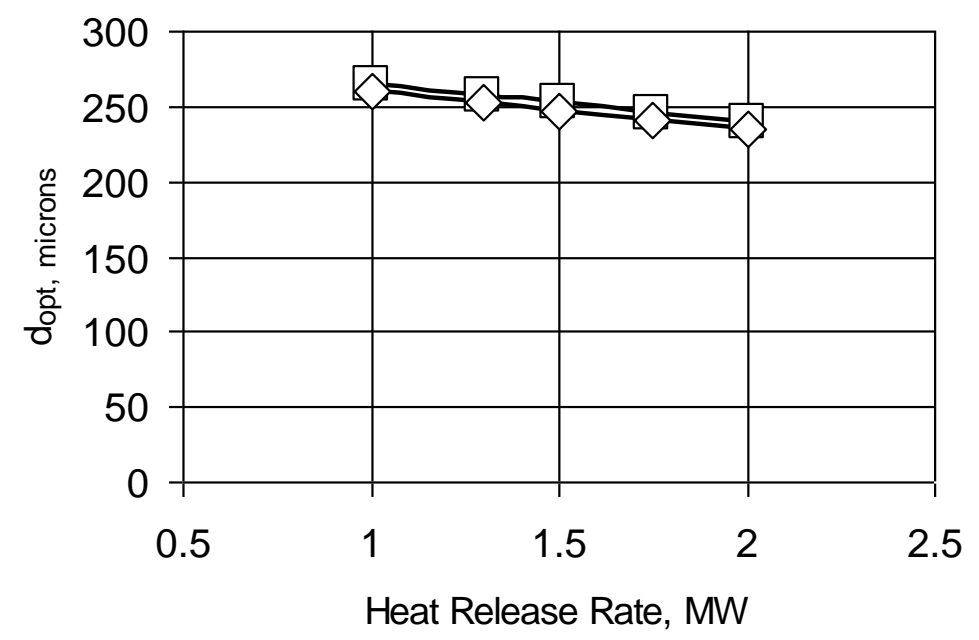

Figure 3 Comparison between optimum droplet diameters predicted by analytical formulae $(16,20)$ and by numerical analysis

$\square$ - analytical; $\diamond$ - numerical

The equation (9) is modified slightly to give

$d^{2} V \frac{d V}{d z}=\alpha(U(z)-V)-d^{2} g$

A $d^{2}$-law of evaporation [14] is a good approximation for the droplet diameter history. Relevance of this approximation for droplets moving in fire plumes can be confirmed, for example, by numerical analysis of the droplet motion.

Specifically, the $d^{2}$-law implies that the droplet surface area diminishes at a constant rate:

$d_{0}^{2}-d^{2}(t)=k_{e v} \cdot t$

which is equivalent to 
$\frac{d}{d z}\left(d^{2}\right)=-\frac{k_{e v}}{V}$

The evaporation constant $k_{e v}$ is estimated as follows [14]

$$
k_{e v}=\frac{8 k_{g}}{\rho_{p} c_{p}} \ln \left(1+\frac{c_{p}}{L}\left(T_{g}-T_{s}\right)\right)
$$

Integration of the equation (21) from the initial point down to the fuel surface gives an approximate relation

$$
-\frac{1}{2} V_{0}^{2} d_{0}^{2}+\frac{k_{e v} V_{0}}{4} z_{\max } \approx \alpha\left[\int_{z_{\max }}^{0} U(z) d z-\int_{z_{\max }}^{0} V(z) d z\right]-g \int_{z_{\max }}^{0} d^{2}(z) d z
$$

(Integration by parts is performed on the LHS, with the relation (23) being taken into account. Further, we made an approximation $\left.\int_{z_{\max }}^{0} V(z) d z \approx \frac{V_{0}}{2} z_{\max }\right)$.

Estimating the last two integrals on the RHS of (25) gives

$$
-\frac{1}{2} V_{0}^{2} d_{0}^{2}+\frac{k_{e v} V_{0}}{4} z_{\max } \approx \alpha \int_{z_{\max }}^{0} U(z) d z-\alpha \frac{V_{0}}{2} z_{\max }-g z_{\max } \frac{d_{0}^{2}}{2}
$$

and, finally

$$
d_{o p t} \approx\left[\frac{2}{\left(V_{0}^{2}-g z_{\max }\right)}\left\{\alpha\left(\frac{V_{0} z_{\max }}{2}+\int_{0}^{z_{\max }} U(z) d z\right)+\frac{k_{e v} V_{0}}{4} z_{\max }\right\}\right]^{1 / 2}
$$

The integrated gas velocity profile for the present case takes the following form

$$
\begin{aligned}
& \int_{z_{\max }}^{0} U(z) d z=\gamma_{1}\left(\frac{g}{c_{p} T_{\infty} \rho_{\infty}}\right)^{1 / 3} \dot{Q}_{c}^{1 / 3}\left[\left(L_{f}-z_{0}\right)^{2 / 3}-\left(z_{\max }-z_{0}\right)^{2 / 3}\right]-\frac{13}{15} U\left(L_{f}\right) L_{f} \approx \\
& \approx\left(\frac{g}{c_{p} T_{\infty} \rho_{\infty}}\right)^{1 / 3} \dot{Q}_{c}^{1 / 3}\left[\gamma_{1}\left[\left(L_{f}-z_{0}\right)^{2 / 3}-\left(z_{\max }-z_{0}\right)^{2 / 3}\right]-\gamma_{3}\left(L_{f}-z_{0}\right)^{-1 / 3} L_{f}\right]
\end{aligned}
$$

$\left(\gamma_{3}=13 / 15 \gamma\right)$ 
Comparison with the numerical integration results are presented in Figure 4 for a range of fire sizes spanning nearly order of magnitude in HRR. The agreement is particularly good for smaller fires $(\sim 1 M W)$ with the optimum diameter starting to be slightly under-predicted for larger fires. Overall, deviation of the analytical estimation from "exact" (numerical) solution does not exceed 13\%.

Of the particular interest is a dependence of an optimum diameter on the fire HRR. The rate of the diameter increase with fire size is easily deducible from (27) in the form

$$
\frac{d}{d \dot{Q}}\left(d^{2}\right) \propto \dot{Q}^{-9 / 30}=\dot{Q}^{-0.3}
$$

In reality, the rate is slightly bigger, as suggested by Figure 4. It is intended to perform more sophisticated analysis of the equation (21) in order to improve predictions.

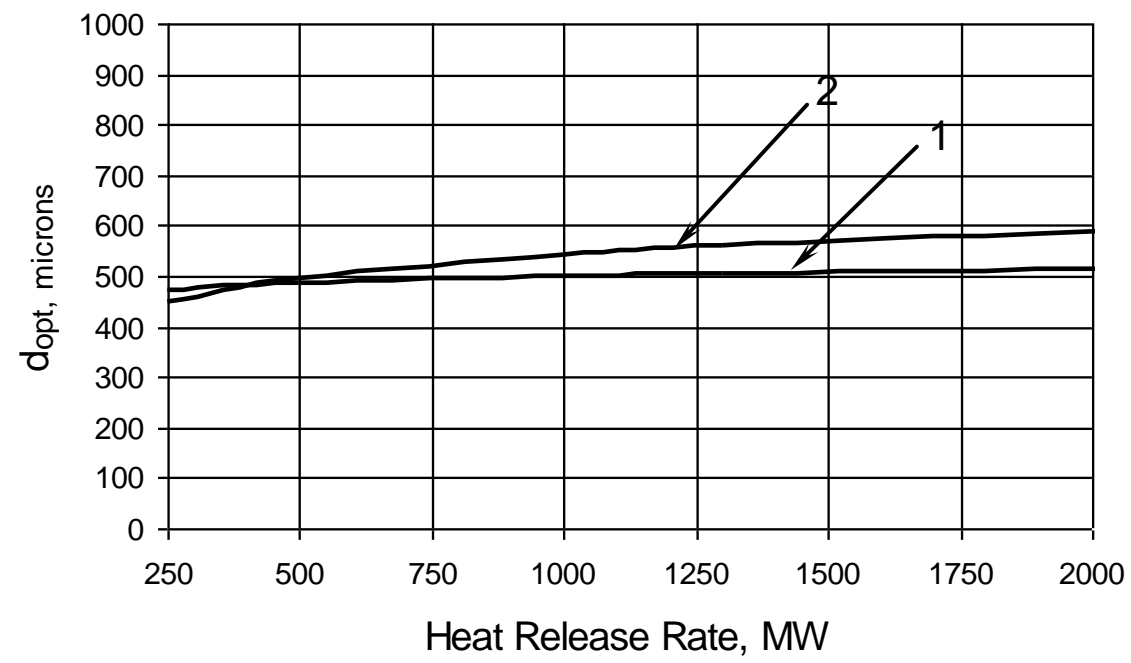

Figure 4 Comparison between optimum droplet diameters predicted by analytical formulae $(27,28)$ and by numerical analysis

1 - analytical; 2 - numerical

The proposed concept of optimum droplet diameter allows scientifically clear definition of water mist to be proposed. At the present, definitions used in the literature are rather arbitrary. One can define water mist spray as such that aims at gaseous flame suppression, rather than surface suppression. In this mode, droplets will evaporate above the surface of the burning material. If such a definition is adopted then it becomes clear that the actual droplet size in water mist spray 
cannot be specified without referring to a particular fire. Indeed, minimum diameter of droplets penetrating through the flame towards surface is a function of fire Heat Release rate (Figure 4). With the help of the estimation of optimum droplet diameter proposed in the present paper, one can quantitatively define water mist spray for a particular fire with a specific HRR as such that droplet diameters in the spray satisfy

$d \leq d_{\text {opt }}(\dot{Q})$

\section{Conclusion}

Equations of droplet motion and evaporation in fire plumes do not admit exact solutions. Despite of this fact, it is possible to derive a range of useful quantitative results in an analytical manner. One possible application of the analysis, demonstrated in the present paper, is the concept of an optimum droplet diameter for fire fighting.

A new concept of an optimum diameter has been proposed, based on the strategy of complete droplet evaporation in the flame. Such definition would be of particular importance for water mist suppression systems, relying on gaseous flame suppression.

The proposed definition of the optimum droplet requires analysis of the droplet motion and evaporation within fire plume and flame. The analysis has been performed using Lagrangian equation of droplet dynamics. Despite the absence of exact solutions, the nature of applied optimum criterion allowed quantitative results to be derived.

An optimum diameter has been predicted analytically for fires in the range from 250 to $2000 \mathrm{~kW}$ to within $13 \%$ of the accurate numerical solution. An optimum diameter has turned out to be a weak function of the fire Heat Release Rate. The form of the latter dependence has been derived.

Results are encouraging in a sense that a rather complicated problem has been solved with a good accuracy by fairly simple considerations. It is planned to perform more sophisticated analysis in order to improve results further.

\section{Notation}

$C_{D}$

$c_{p}$ drag coefficient

gas specific heat 
$d$

$d_{0}$

$d_{\text {opt }}$

$\vec{F}_{g}$

$g$

$k_{e v}$

$k_{g}$

$L$

$L_{f}$

$m$

$\dot{Q}$

$\dot{Q_{c}}$

$\operatorname{Re}_{p}$

$T_{\infty}$

$T_{g}$

$T_{s}$

$t$

$U$

V

$V_{0}$

$z$

$z_{\max }$

$z_{\text {min }}$

$z_{0}$

Greek symbols

$v$

$\rho$

$\rho_{p}$

$\rho_{\infty}$ droplet diameter

initial droplet diameter

optimum droplet diameter

gravity force

gravity acceleration

evaporation constant

gas thermal conductivity

latent heat of vaporization

mean flame height

droplet mass

fire Heat Release Rate

fire convective Heat Release Rate

particle (droplet) Reynolds number

ambient temperature

gas temperature

droplet surface temperature

time

fire plume velocity

droplet velocity

initial droplet velocity

height

initial droplet height

bottom point of droplet trajectory

fire plume virtual origin

gas kinematic viscosity

gas density

particle (droplet) density

ambient density

\section{References}

[1] Morsi, S.A. and Alexander, A.J., "An Investigation of Particle Trajectories in Two-phase Flow Systems", Journal of Fluid Mechanics, Vol. 55, (1972), pp. 193-208. 
[2] Novozhilov, V., "On some Integrable Cases of Particle Motion in a Fluid", Mathematics in Engineering, Science and Aerospace, Vol. 1, No. 4, (2010), pp. 371-380.

[3] Novozhilov, V., Harvie, D.J.E., Green, A.R. and Kent, J.H., "A Computational Fluid Dynamics Model of Fire Burning Rate and Extinction by Water Sprinkler", Combustion Science and Technology, Vol. 123, No. 1-6, (1997), pp. 227-245.

[4] Novozhilov, V., Harvie, D.J.E., Kent, J.H., Apte, V.B. and Person, D., “A Computational Fluid Dynamics Study of Wood Fire Extinguishment by Water Sprinkler“, Fire Safety Journal, Vol. 29, No. 4, (1997), pp. 259-282.

[5] Novozhilov, V., "Fundamentals and Application of Fire Suppression Modeling", Proceedings of the Second NRIFD Symposium - Science, Technology and Standards for Fire Suppression Systems, (2002), pp. 195215.

[6] Grant, G., Brenton, J. and Drysdale, D., "Fire Suppression by Water Sprays", Progress in Energy and Combustion Science, Vol. 26, (2000), pp. 79-130.

[7] Herterich, O., Water as an Extinguishing Agent, Alfred Hüthig Publishing Company, (1960).

[8] Ohkubo, H. and Nishio, S., "Study on Accurate Prediction of Heat Transfer Characteristics of Mist Cooling", International Journal of Japanese Society of Mechanical Engineers, Series II, Vol. 33, No. 2, pp. 326-332.

[9] Kaleta, A., "Effect of Drop Size on Extinguishing Effectiveness of a Water Spray", Archiwum Combustionis, Vol. 6, No. 3-4, (1986), pp. 201- 212.

[10] Kuo, K.K., Principles of Combustion, Wiley, (2005).

[11] Dickerson, R.A. and Schuman, M. D., Journal of Spacecraft, (1960), p. 99.

[12] Polyanin, A.D., Zaitsev, V.F., Exact Solutions for Ordinary Differential Equations (2nd ed.), Chapman \& Hall/CRC, (2003).

[13] Karlsson, B. and Quintiere, J.G., Enclosure Fire Dynamics, CRC Press, (2003).

[14] Lefebvre, A., Atomization and Sprays, Hemisphere, (1989). 